\title{
Patrones VALORATIVOS DE ACTITUD EN ANOTACIONES EN EL LIBRO DE CLASES: JUICIOS, AFECTOS Y APRECIACIONES DE
LOS DOCENTE EN UN CONTEXTO EDUCATIVO*
}

\author{
Marco Antonio Alarcón**
}

\section{Resumen}

El artículo analiza algunos patrones valorativos de actitud encontrados en las anotaciones de un libro de clases de un liceo municipal, a partir de la selección de 174 anotaciones, correspondientes al año 2005 de un noveno grado de educación, (actualmente, primer año medio de secundaria en Chile). Las anotaciones sobre las conductas de los estudiantes pueden incidir en su continuidad o exclusión en un establecimiento educativo. Este trabajo se basa en los enfoques complementarios de la LSF y el ACD, y se centra en el análisis de la Valoración (White, 1999, Martin, 2000; Martín y Rose, 2003; Martin y White, 2005) en particular, desde el subsistema de actitud considerando los parámetros de juicio, afecto y apreciación. El análisis permite crear una red de significados e identificar ciertas funciones y/o propósitos de los docentes al producir las anotaciones.

Palabras clave: Modelo de la Valoración, Subsistema de actitud, Anotaciones en el libro de clases, Discurso profesional docente.

\section{EVALUATIVE ATTITUDE IN SCORING PATTERNS IN THE BOOK CLASS: TRIALS, EMOTIONS AND INSIGHTS OF TEACHERS A VULNERABLE EDUCATIONAL CONTEXT}

\begin{abstract}
The article discusses some evaluative attitude patterns found in the annotations of a book by a municipal high school classes, from the selection of 174 entries, for the year 2005 of a ninth grade education, (now first half year in middle school Chile). The annotations on student behaviors may influence its continuation or exclusion in an educational establishment. This work is based on complementary approaches of SFL and CDA, and focuses on the analysis of the Rating (White, 1999, Martin, 2000; Martin and Rose, 2003; Martin and White, 2005) in particular, from the subsystem attitude considering the parameters of judgment, affection and appreciation. The analysis allows to create a network of meanings and identify certain functions and / or purposes of teachers to produce annotations.
\end{abstract}

Keywords: Valuation Model, Subsystem attitude, Annotations on the Book class, teacher professional discourse.

Recibido: 15-08- $2014 \quad$ Aceptado: 07-01-2015

* Investigación correspondiente al Proyecto Semilla UCSH FSEM13-08 Patrones valorativos de actitud en el discurso profesional docente: juicios, afectos y apreciaciones de los docentes en un contexto educativo vulnerable.

** Chileno, Académico Asociado del Departamento de Castellano de la Universidad Metropolitana de Ciencias de la Educación, UMCE. Doctor (C) en Lingüística por la Universidad Católica de Chile, maalarcon@uc.cl 


\section{Introducción}

En este trabajo analizamos algunos patrones evaluativos empleados en las anotaciones en el libro de clases registradas por los profesores de primer año medio de un liceo municipal de una comuna del sector poniente de la Región Metropolitana. El propósito de las anotaciones en el libro de clases es describir el comportamiento de los alumnos en relación al cumplimiento y/o incumplimiento de las normas y reglamentos. Dichas normas se amparan en un ethos supuestamente compartido por toda la comunidad escolar, incluyendo profesores y directivos, apoderados y estudiantes. Sin embargo, nos encontramos con valoraciones (evocadas e inscritas) que dan cuenta de las emociones y afectos personales tanto de los docentes hacia los estudiantes, como de los estudiantes hacia los docentes. Asimismo, estas anotaciones revelan el interés/desinterés que tienen los estudiantes hacia los procesos de aprendizaje.

Este trabajo está motivado por el interés en comprender cómo se manifiesta la valoración en las anotaciones del Libro de clases de un Liceo vulnerable en la relación interpersonal profesor-alumno desde el subsistema de actitud: juicio, afecto y apreciación del Modelo de la Valoración. Estas valoraciones expuestas en las anotaciones en el libro de clases tienen el potencial de evidenciar como se asignan roles, prejuicios y creencias específicas de parte de los docentes, hacia los estudiantes, las cuales inciden en la permanencia y / o exclusión de estos jóvenes vulnerables en el colegio, y en última instancia, dentro del sistema educativo.

Desde la perspectiva del Análisis Crítico del Discurso (ACD), se ha hechouna escasa investigación que contempleun análisisléxico-gramatical detallado de la interacción profesor-alumno. Las investigaciones existentes desde el ACD, asociadas a valoración y contexto escolar, se han centrado en la descripción de valoraciones del discurso docente en libros de texto (Achugar, 2008; Oteíza, 2006, 2009; Moyano, 2010). Por tanto, el foco de los estudios ha estado centrado en textos que involucran la transferencia de conocimientos hacia los estudiantes y no la interacción directa entre profesor y alumno en la sala de clases. Por ello, consideramos necesario realizar un estudio que involucre dicha interacción no abordada. Esto puede cumplirse a partir del análisis del género anotación en el libro de clases. Este estudio pretende dar cuenta de la interacción profesor-alumno en la sala de clases, a través del análisis de los patrones valorativos presentes en las anotaciones escritas por los docentes. 
Este estudio, inserto en la práctica social escolar, tiene el potencial de contribuir a la descripción de la manifestación de las relaciones de control y de poder desde el rol docente, considerando que las anotaciones las ejecuta sólo el docente y/o docente directivo, y en ningún caso los estudiantes. Por tanto, el registro se escribe desde una posición única. Ello da cuenta de las desigualdades e inequidades de la construcción de anotaciones, lo cual obedece a la constitución del sistema escolar que no considera la participación de sus diversos actores en la construcción de sus reglamentaciones y normas (Casas et al., 2008). No obstante, en la actualidad se espera que los sistemas escolares sean democráticos, no sólo en la construcción participativa de sus reglamentos y normas, sino también en la aplicación de sanciones, por tanto, consideramos que el presente estudio será un aporte para la construcción de un sistema de relaciones democráticas al interior de la escuela, puesto que a través de nuestro análisis se revelan las inequidades de participación y valoración existentes en el actual sistema. En dicho sentido las valoraciones asociadas positiva o negativamente al cumplimiento y/o incumplimiento de reglas y/o conductas esperadas o deseadas desde el sistema escolar, deben estar insertas en un nivel social acorde a los valores y leyes que sustentan la democracia, el respeto, la tolerancia, la no discriminación y la valoración positiva de las diversas minorías sociales, culturales, sexuales, étnicas y religiosas, ley ํ⒛536 (BCN, 2011). Además, deben ser coherentes con los diversos principios y tratados suscritos a nivel nacional e internacional, coincidiendo con los principios de derechos humanos, derechos del niño, derechos de la mujer, derechos étnicos, libertad religiosa, derechos sexuales, entre otros.

Este trabajo se organiza en cuatro secciones. Primero comenzaremos con una breve discusión sobre el modelo de la Valoración dentro de la Lingüística Sistémico Funcional (LSF) y el Análisis Crítico del Discurso (ACD), planteando que el modelo de la Valoración (Appraisal Theory) puede utilizarse como una poderosa herramienta teórica y metodológica para la comprensión de las relaciones interpersonales a nivel escolar, principalmente entre profesores y estudiantes, al focalizarse en el funcionamiento retórico de los recursos valorativos en el plano discursivosemántico. En segundo lugar nos referiremos muy brevemente, al contexto de producción de las anotaciones en el libro de clases y su función o mecanismo de exclusión dentro del sistema escolar. Tercero, presentamos el análisis de las anotaciones desde el subsistema de actitud, consideran- 
do los parámetros de juicio, afecto y apreciación, respectivamente. Ello con el objetivo de mostrar los diferentes recursos lingüísticos que operan a nivel individual en cada parámetro, y a su vez, de forma combinada creando patrones de valoración de reiteración principalmente negativa que justifiquen la sanción y la exclusión de los estudiantes, entre otros patrones. Este análisis se organiza a su vez en tres etapas. Primero se presentan las tres dimensiones de patrones valorativos, la primera referida a reglamentación de la permanencia que da cuenta de patrones que valoran personas, conductas, procesos y productos referidos a los rendimientos académicos, la disciplina, los procedimientos ante conductas y la toma de conocimiento del apoderado; la segunda referida la presentación personal que da cuenta de patrones valorativos referidos al uso del uniforme escolar y al uso de accesorios, y la tercera, referida a la valoración de la labor académica docente que da cuenta de la colaboración de los alumnos en dicha acción.

Finalmente, mostramos a modo de síntesis la distribución por parámetros de actitud del corpus de anotaciones analizado, y las conclusiones de la distribución por patrones valorativos de las anotaciones analizadas puntualizando cómo los recursos léxico-gramaticales específicos funcionan en conjunto para expresar al intensificación de la necesidad de exclusión de los estudiantes del establecimiento, lo que incluso puede llegar a su salida del sistema escolar. Con este análisis proponemos una comprensión de la interacción profesor-alumno en un contexto escolar empírico.

\section{Marco teórico}

\section{LSF y Modelo de la Valoración}

Dentro del marco de la lingüística sistémico-funcional (en adelante LSF) desarrollada por Halliday (1994), el modelo de la Valoración corresponde a un novedoso instrumento teórico y de análisis para abordar el estudio de la evaluación del lenguaje (White, 1998; Martin, 2000; Martin y Rose, 2003; Martin y White, 2005). Desde dicho enfoque se desarrolla el significado interpersonal y se describe el modo en que se usa el lenguaje para evaluar, ya sea, por medio de la creación de personas textuales, la construcción de posturas y/o posiciones determinadas, o bien, por medio del manejo de las relaciones interpersonales. Este modelo aborda la 
expresión de actitud que da cuenta de sentimientos, creencias y valores en diversos contextos comunicativos (Kaplan, 2004; Gonzales, 2012).

El modelo de la valoración se desarrolló a partir de la década de 1990 en la Universidad de Sidney, Australia, dentro de un proyecto de investigación educativa realizado principalmente por James Martin, Peter White y David Rose. Entre las conclusiones de su estudio reconocieron la necesidad de desarrollar una semántica interpersonal mucho más fina que la que había planteado el propio Halliday (1994) con el sistema de Modo; de igual forma se requería considerar tanto los efectos retóricos y el posicionamiento social vinculados a las opciones léxico-gramaticales, y la identificación de solidaridad valorativa a partir de la que variarían los elementos interpersonales del contexto social.

En consideración de la LSF el lenguaje corresponde a un recurso sistemático para expresar significados en contexto, es decir, es un sistema de opciones semánticas que los emisores seleccionan para transmitir sus mensajes a determinados receptores. Dichas opciones son dependientes del contexto y están registradas en tres niveles: el fonológico, el léxico-gramatical y el semántico. Dichos planos del significado denominados metafunciones operan de forma simultánea y se refieren al contenido, a la interacción y al texto, los que se corresponden con las metafunciones ideacional, interpersonal y textual, respectivamente. Justamente, el modelo de la Valoración se ocupa de especificar la semántica de la metafunción interpersonal en su interacción social y se refiere a la expresión valorativa de la actitud hacia personas, acciones, eventos, procesos y productos.

\section{El modelo de la valoración}

Entendemos por valoración (Appraisal) la construcción discursiva de la actitud y de la postura intersubjetiva. Comprende, entonces, todos los usos de carácter evaluativo disponibles en las lenguas. Sin embargo, el alcance de la valoración es mayor, puesto que también incluye la negociación de estas evaluaciones, que los hablantes llevan a cabo en sus interacciones comunicativas con receptores reales o potenciales. De acuerdo con la definición anterior, la valoración cumple con funciones específicas, que se relacionan con su carácter discursivo y evaluativo. Estas funciones implican el establecimiento de un posicionamiento actitudinal, un posicionamiento dialógico y uno intertextual. 
El posicionamiento intertextual puede comprenderse como una subcategoría en el posicionamiento dialogístico, ya que, desde este modelo corresponde a una ubicación dialógica y actitudinal, entendida como un modo de posición evaluativa. Así, estos usos del lenguaje permiten adoptar posturas evaluativas respecto de las proposiciones enunciadas, dichas evaluaciones pueden provenir de diversas fuentes externas, incluyendo otros sujetos. La construcción básica es la referencia a las palabras de otro, que dan cuenta de una relevancia que implica una atribución como propósito en la interacción presente en que se enuncia como actualización.

\section{Los subsistemas de la valoración}

Los recursos de evaluación operan en tres subsistemas o dominios semánticos: ACTITUD, COMPROMISO y GRADACIÓN.

En la actitud operan los significados en que los hablantes atribuyen una evaluación intersubjetiva sobre participantes y/o procesos. Tales valoraciones responden a respuestas emocionales que pueden estar asociadas a patrones de valores validados culturalmente. El compromiso se refiere a ciertos recursos que pueden ser utilizados para posicionar la voz, ya sea del hablante o del autor en vinculación con distintas proposiciones planteadas en el texto. Finalmente, a través de la gradación se gradúa semánticamente la intensidad en que los hablantes valoran sus enunciados (White, 1999; Martin y White, 2005).

\section{Figura 1}

Sistema de la valoración

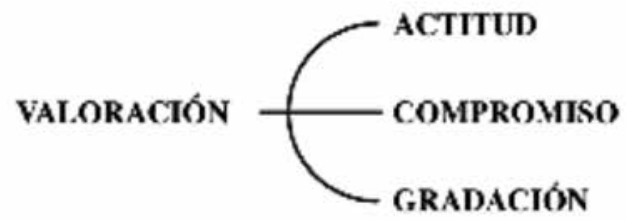




\section{La actitud}

Se consideran actitudinales los enunciados que incorporan una evaluación positiva o negativa, o que pueden interpretarse como evaluación indirecta o evocada que puede hacer un lector a partir de un enunciado. Esta categoría se constituye, en tres parámetros, AFECTO, JUICIO, y APRECIACIÓN. El afecto corresponde a la descripción de relaciones emocionales; el juicio apunta a la evaluación de acciones humanas en relación a las normas sociales establecidas y aporta un sentido ético y moral de la conducta; y la apreciación se refiere a la evaluación de productos, objetos, procesos o construcciones textuales desde una perspectiva estética y de valoración social. No obstante, las categorizaciones no son absolutas sino que dependen de la cultura en que está enraizado el análisis. El afecto, corresponde según White (2004), a la categoría básica de actitud, de la cual, según a qué sujeto u objeto se evalúa deriva en las otras categorías antes señaladas. Este subsistema de actitud permite evaluar y controlar el comportamiento humano, además de incorporar la apreciación de las acciones y del entorno natural o cultural.

Figura 2. Relaciones entre los subsistemas de actitud (Tomado de Kaplan, 2004, quien lo adaptó de Martin 2000)

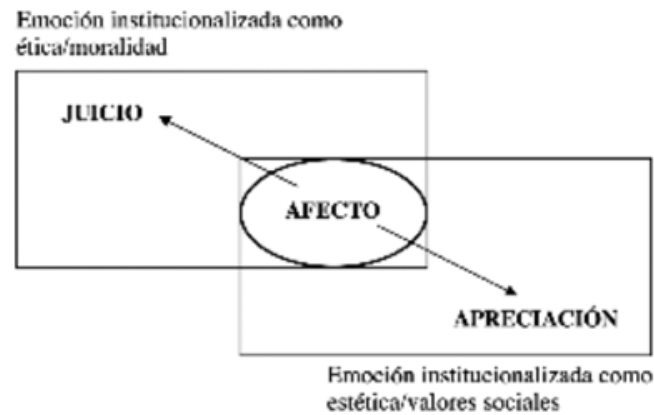

La actitud puede textualizarse de forma explícita o implícita. A nivel explícito se transmite un sentido positivo o negativo de forma directa. A nivel implícito, el análisis se complejiza, ya que depende de la interpretación que le otorgue el lector, desde sus creencias y/o expectativas. Por esta razón, las actitudes no dependen de palabras específicas, sino de la articulación textual y contextual en que se generen dentro de una interacción textual humana. 


\section{El juicio}

El juicio evaluativo corresponde a la institucionalización de las emociones dentro de un contexto normativo y regulatorio. En función de lo que se debe o no deber hacer. De tal forma, a través del juicio es posible calificar las conductas como morales o inmorales, legales o ilegales, socialmente válidas, o inaceptables, normales o anormales, etc. A partir de esto, el juicio puede categorizarse en dos tipos: (i) JUICIOS DE ESTIMA SOCIAL, dividido, a su vez, en juicios relativos a la normalidad, la capacidad o la tenacidad demostrada en la conducta; y (ii) JUICIOS DE SANCIÓN SOCIAL, vinculados con la verdad y la integridad moral.

Por cierto, el parámetro de juicio depende del contexto cultural e ideológico específico en que ocurre. La forma que las personas juzgan la moralidad, la legalidad, la capacidad u otras características conductuales humanas dependen de la cultura, y de la relación que tienen con las creencias y experiencias personales. De tal manera que siempre existe la posibilidad que un mismo hecho sea valorado con juicios diferentes, dependiendo de la posición ideológica del evaluador.

Figura 3. Parámetros de juicio adaptación de Kaplan (2004) a partir de Martin (2000)

\begin{tabular}{|c|c|c|}
\hline \multicolumn{3}{|c|}{ JUICIO } \\
\hline & $\begin{array}{l}\text { ESTIMA SOCIAL } \\
\text { E.jemplos positivos } \\
\text { (adminación) }\end{array}$ & $\begin{array}{l}\text { Ejcmplos negativos (crítica, } \\
\text { sin implicaciunes legales) }\end{array}$ \\
\hline normalidad & $\begin{array}{l}\text { corriente, común, normal, } \\
\text { afortunado'a, moderno/a }\end{array}$ & $\begin{array}{l}\text { excéntrico/a, extrano/a, raro/a, } \\
\text { desafortunado/a, anticuado/a }\end{array}$ \\
\hline capacidad & $\begin{array}{l}\text { habilidoss'a, inteligente, } \\
\text { intuitivo'a, atlético'a, fuerte }\end{array}$ & $\begin{array}{l}\text { inhábil, lentìa, tonts'a, torpe, } \\
\text { débil }\end{array}$ \\
\hline \multirow[t]{2}{*}{ tenacidad } & $\begin{array}{l}\text { heroico'a, valiente. confiable, } \\
\text { infatigable, perseverante }\end{array}$ & $\begin{array}{l}\text { cobarde, apresurado'a. no } \\
\text { confiable, distraído'a, perezoso/a }\end{array}$ \\
\hline & $\begin{array}{l}\text { SANCIÓN SOCIAI. } \\
\text { Ejemplos positivos } \\
\text { (alahamza) }\end{array}$ & $\begin{array}{l}\text { Ejemples negativos (condena, } \\
\text { puede lener implicacicies legales) }\end{array}$ \\
\hline veracidad & $\begin{array}{l}\text { sincero'a, honesto/a, genuino'a, } \\
\text { francis'a, directo/a }\end{array}$ & $\begin{array}{l}\text { deshonestofa, mentirosola, } \\
\text { inauténticofa, manipulador/a }\end{array}$ \\
\hline $\begin{array}{l}\text { integridad } \\
\text { moral }\end{array}$ & $\begin{array}{l}\text { moral, hondadosoin, respetuonolía } \\
\text { de la ley, sensible. justo/a }\end{array}$ & $\begin{array}{l}\text { inmoral, malvado'a, comupto/a, } \\
\text { cruel, injusto'a }\end{array}$ \\
\hline
\end{tabular}


Los juicios se expresan explícitamente por medio de adverbios, atributos y epítetos, sustantivos y verbos. No obstante, muchas veces el juicio puede figurar implícitamente, o ser evocado de manera indirecta a través de indicadores (tokens) de juicio. Así, una descripción de tipo factual en apariencia, puede incluir el juicio de un hecho, situación, persona o grupo. Si alguien señala que el actual gobierno no previno los atochamientos en la salida de fin de semana largo, puede estarse refiriendo indirectamente a que el gobierno es inepto. La potencialidad de estos significados depende de la ideología de quien lo produce y de quien lo interpreta.

\section{El afecto}

El afecto corresponde a la evaluación asociada a respuestas emocionales, propias o ajenas, hacia personas, cosas o eventos (White, 2004). Dichas respuestas se organizan en tres grupos polares, primero felici$\mathrm{dad} /$ infelicidad, segundo seguridad/inseguridad y tercero satisfacción/ insatisfacción. La manifestación de afecto puede expresarse por medio de (i) una cualidad, con el uso de un epíteto para describir a los participantes, (ej.: Una persona amable); un atributo (ej. La mujer era amable); o una circunstancia (ej. La mujer escuchó amablemente); (ii) un proceso (ej.: afectivo mental: El ipad a bajo precio interesó al profesor; afectivo conductual: El profesor frunció el ceño); o (iii) un comentario (ej.: la marcha terminó tristemente).

Figura 4. Las categorías correspondientes al subsistema de afecto se resumen en la Tabla tomada de Kaplan (2007).

\begin{tabular}{|lll|}
\hline \multicolumn{1}{|c|}{ Categoría } & \multicolumn{1}{c|}{$\begin{array}{c}\text { AFECTO } \\
\text { Ejemplos positivos }\end{array}$} & \multicolumn{1}{c|}{ Ejemplos negativos } \\
\hline felicidad/infelicidad & $\begin{array}{l}\text { feliz, alegre, jubiloso/a, } \\
\text { optimista }\end{array}$ & $\begin{array}{l}\text { deprimido/a, triste, } \\
\text { miserable, angustiado/a }\end{array}$ \\
\hline seguridad/inseguridad & $\begin{array}{l}\text { confiado/a, seguro/a, } \\
\text { tranquilo/a, sereno/a }\end{array}$ & $\begin{array}{l}\text { ansioso/a, preocupado/a, } \\
\text { inseguro/a, intranquilo/a }\end{array}$ \\
\hline satisfacción/insatisfacción & $\begin{array}{l}\text { interesado/a, absorto/a, } \\
\text { estar enfrascado/a, gustar }\end{array}$ & $\begin{array}{l}\text { cansado/a, aburrido/a, } \\
\text { exasperad/o, odiar }\end{array}$ \\
\hline
\end{tabular}

Entre los marcadores de afecto se cuentan verbos de emoción, que dan cuenta de procesos mentales (ej.: amar/odiar); adverbios que describen circunstancias de modo (ej.: alegremente/tristemente); adjetivos formulan emoción (ej.: feliz/triste), y nominalizaciones, correspondientes a modificaciones de verbos y adjetivos en sustantivos (ej.: alegría/ desesperación). 
El afecto se entiende como autoral si se construye en primera persona, y no-autoral si va en segunda o tercera persona. El caso autoral indica la respuesta emocional personal, y por ende, implica la responsabilización de dicha emoción. En el caso del afecto no autoral, se describe la emoción de un tercero, y por ende, la responsabilidad de la emoción no corresponde al hablante. En suma la expectativa de la expresión de afecto del hablante en uno y otro caso, implica la necesidad de aceptación y solidaridad de parte del oyente. Un factor relevante para el logro o desacierto del hablante es la confiabilidad o cercanía que pueda establecer con el oyente o destinatario, ello se implica a su vez con el rol social o autoridad que tenga el hablante en relación a sus destinatarios. Esto se evidencia, por ejemplo, en el rol que cumplen personajes de la socialité de un país que pasan a ser rostros de campañas publicitarias, ya que, la empatía establecida previamente con el público es finalmente lo que permite la confiabilidad de sus enunciados, y es por cierto, la razón por la cual fue escogido como rostro de una determinada campaña. Así, su confiabilidad genera una legitimidad afectiva.

Según Martin (2000), para clasificar el afecto, deben tomarse en cuenta las siguientes variables: (i) si los sentimientos son construidos como positivos o negativos por la cultura; (ii) si los sentimientos involucran una manifestación paralingüística (ej.: sudar), o si se experimenta como un estado mental (ej.: discreto); (iii) si los sentimientos se producen como reacción a un agente externo determinado (ej. Al profesor le encantaban sus alumnos), o como un estado sin una dirección determinada (ej. La abuela estuvo contenta); (iv) cómo se regulan o escalan los sentimientos, (ej.: bajo: A Juan le agradó la sorpresa / medio: A Juan le gustó la sorpresa / alto: A Juan le fascinó la sorpresa); y, en definitivas, (v) si las emociones operan intencionalmente frente a un estímulo "realis", es decir, vinculado con estados presentes (ej. Al profesor le disgustó la broma), o "irrealis", vinculado con estados futuros, (ej. Al profesor le encantaría salir de paseo con el curso).

\section{La apreciación}

La apreciación corresponde a las evaluaciones sobre producciones, objetos, procesos o entidades. A su vez, es posible evaluar personas, no obstante requiere que sean entendidas como entidades y no como participantes. La evaluación apreciativa da cuenta de características estéticas y sociales; los aspectos estéticos se refieren a constitución, composición e impacto, y el nivel estético se refiere a la relevancia social. 
Con estos valores se evalúan la forma, la apariencia, la composición, el impacto y la importancia social. Este sistema incluye, por lo tanto, una evaluación estética (ej. una linda mañana, una presentación estupenda, una obra sensacional) así como una categoría de valuación social, no estética (ej. un argumento conciliador, una idea prioritaria).

Martin (2000) categoriza la apreciación alrededor de tres dimensiones: (i) REACCIÓN, (ii) COMPOSICIÓN, y (iii) VALUACIÓN. La reacción describe el nivel de atracción atencional y el impacto provocado por el objeto, proceso o texto evaluado. La composición se vincula con la percepción de proporción y detalle; y la valuación, se relaciona con el valor social de lo evaluado. Por su parte, la reacción se divide en valores de impacto y calidad, y la composición, en valores de balance y complejidad.

Existen varias relaciones de semejanza y de diferencia entre la apreciación y el juicio. Ambas se orientan a lo evaluado, por sobre el evaluador. Siendo menos personalizadas que el afecto, que tiende a ser mayormente subjetivo. Así, el juicio se focaliza en las conductas humanas, la aprecia-

Figura 5. Adaptada de Martin (2000), muestra las categorías y sub-categorías de la apreciación.

\begin{tabular}{|c|c|c|}
\hline \multicolumn{3}{|c|}{ APRECIACIÓN } \\
\hline & Positiva & Negativa \\
\hline $\begin{array}{l}\text { REACCIÓN: } \\
\text { Impacto }\end{array}$ & $\begin{array}{l}\text { cautivador/a, llamativo/a, atractivo/a, } \\
\text { agradable, conmovedor/a }\end{array}$ & $\begin{array}{l}\text { aburrido/a, tedioso/a, ascético/a, } \\
\text { pedante, soso/a }\end{array}$ \\
\hline $\begin{array}{l}\text { REACCIÓN: } \\
\text { Calidad }\end{array}$ & $\begin{array}{l}\text { hermoso/a, espléndido/a, } \\
\text { cncantador/a }\end{array}$ & feo/a, repulsivo/a, repugnante \\
\hline $\begin{array}{l}\text { COMH'OSICIÓN: } \\
\text { Balance }\end{array}$ & $\begin{array}{l}\text { balanceado/a, armonioso/a, } \\
\text { simétrico/a, proporcionado/a }\end{array}$ & $\begin{array}{l}\text { desbalanceado/a, discordante, } \\
\text { desproporcionado/a, asimétrico/a }\end{array}$ \\
\hline $\begin{array}{l}\text { COMPOSICIÓN: } \\
\text { Complejidad }\end{array}$ & $\begin{array}{l}\text { simple, elegante, detallacio/a, } \\
\text { preciso/a, intrincado/a }\end{array}$ & $\begin{array}{l}\text { extravagante, monolítico/a, } \\
\text { simplista, impreciso/a }\end{array}$ \\
\hline VALUACIÓN & $\begin{array}{l}\text { profundo/a, innovador/a, original, } \\
\text { único/a. exigente }\end{array}$ & $\begin{array}{l}\text { superficial, insignificante, } \\
\text { reaccionario/a. conservador/a }\end{array}$ \\
\hline
\end{tabular}

ción incluye además objetos y productos concretos y abstractos. Dado que las acciones humanas involucran conciencia, voluntad e intencionalidad, el juicio se determina polarmente entre bueno y malo, correcto e incorrecto, atribuyendo responsabilidad o culpa al participante, lo cual es imposible en el caso de la apreciación. Desde esta misma perspectiva Martin (2000) señala que las dimensiones de la apreciación se vinculan a la variable de campo, por lo que invita a que los analistas expliciten su posición como lectores. Finalmente, el análisis de los valores del sistema de actitud requiere su aplicación prosódica, considerando que los patrones valorativos se despliegan acumulativamente en el texto. Se requiere además, considerar el contexto, ya que suele utilizarse como estrategia 
de negociación interpersonal la combinación de evaluación directa y evocada para que el destinatario termine compartiendo sus interpretaciones de actitud.

\section{Práctica social de las anotaciones en el libro de clases}

En esta sección se ofrece una breve presentación contextual que permite situar el análisis de las anotaciones en el libro de clases. Estas anotaciones se basan en el registro escrito que realizan los docentes y/o docentes directivos sobre las conductas relevantes y/o destacadas que implican cumplimiento/incumplimiento de las normas, reglamentos escolares y / o manuales de convivencia del establecimiento por parte de los estudiantes. En el libro de clases existe la sección de ficha personal para cada alumno/a, dicho apartado incluye el espacio para una fotografía tamaño carné, y el espacio para escribir la anotación, considerando la fecha y la asignatura de la anotación. Además, existe una ficha grupal para las anotaciones que involucran al curso completo. La recomendación del uso de la anotación implica haber agotado primeramente el recurso de la amonestación verbal, y en el caso de la reiteración de la conducta indebida, se debiera utilizar la anotación escrita, ello en el caso particular de la anotación negativa. La producción de las anotaciones se realiza regularmente dentro de la sala de clases y en virtud de una acción ejecutada por el/la estudiante que el docente considera como relevante de ser registrada, y que mayoritariamente corresponde a una acción indebida. El registro de anotaciones y su acumulación, así como el procedimiento de sanción gradual debe estar estipulado en los reglamentos escolares. Los lectores obligados de estas anotaciones a nivel escolar son el inspector general, el profesor jefe y el director del establecimiento. El inspector debe velar por el cumplimiento de las normas del establecimiento, por lo tanto, debe revisar las anotaciones y citar al apoderado del alumno infractor, según la gravedad, o la reiteración de la falta; o bien, si procede una sanción mayor, como suspensión o expulsión. El profesor jefe debe estar informado de las conductas de sus alumnos, por tanto, debe revisar regularmente las anotaciones de los alumnos de su curso. El director debe involucrarse si la sanción es más relevante. De igual forma, los apoderados pueden ser citados por cualquiera de los agentes escolares antes señalados, ya sea para conocer la situación conductual de su pupilo, o inclusive si el alumno/a está en peligro de repitencia. En estas 
entrevistas el profesor jefe, el inspector o el director, según sea el caso, atienden al apoderado y le presentan para su conocimiento las anotaciones y las notas del alumno si corresponde. En dicho sentido la anotación cumple un rol relevante para el control disciplinario y académico. Además, siendo el libro de clases un documento público, las anotaciones no pueden ser borradas o alteradas por ningún miembro de la comunidad escolar, ya que ello constituiría un delito. Actualmente las anotaciones deben basarse en el cumplimiento del reglamento escolar y/o manual de convivencia, la normativa que genera esta condición vinculante es reciente. Por ejemplo, durante la dictadura militar (1973-1990) se podía restringir y discriminar arbitrariamente a los estudiantes con expulsión del establecimiento en cualquier fecha del año. Por ello, a partir de la llegada de la democracia (1990) el Ministerio de Educación (en adelante MINEDUC) realizó importantes modificaciones a fin de evitar procedimientos arbitrarios y expulsiones discriminatorias (Casas et al., 2008). Ahora, si bien existen mejores oportunidades educativas que hace 20 años atrás, aún en los contextos vulnerables, como en el que fueron recogidas las anotaciones que se analizan en este artículo, la prioridad de los jóvenes no está en los resultados académicos. Por lo cual, la labor docente se hace más compleja. Por ende, y en consideración de la complejidad que se presenta para los docentes es posible que estas anotaciones sean uno de los pocos canales de expresión con que cuentan los profesores ante las dificultades de manejo y control normativo. Desde esta misma óptica normativa, las relaciones de poder y control amparados en las anotaciones tienen por finalidad legitimar la acción educativa de los docentes, y a su vez, deslegitimar las acciones conductuales negativas referidas a rendimiento y disciplina que realizan los estudiantes. De igual forma, las anotaciones dan cuenta de una parcialidad del hecho educativo, desde la visión del profesor que la escribe, ya que no se considera la versión y / o interpretación de los hechos relatados en la anotación por parte de los estudiantes. Así, el docente, presenta un doble vínculo, juzga y es parte interesada. No obstante, puede arbitrar bien en el caso de que el conflicto sea entre estudiantes, no ocurre lo mismo, si el conflicto es entre docentes y estudiantes. Por tanto, los profesores escriben las anotaciones y expresan su subjetividad en relación al cumplimiento y/o incumplimiento de las normas que deben llevar a cabo los estudiantes, pero a su vez, reflejan también la presencia del ethos de cada profesor al interpretar las acciones de los estudiantes desde sus juicios, afectos y 
apreciaciones que marcan la actitud de sus valoraciones sobre las conductas, productos y los propios estudiantes.

\section{Análisis y discusión}

Para el análisis presentamos una selección representativa de anotaciones en el libro de clases en las que es posible apreciar cómo los docentes organizan valoraciones en torno a los estudiantes, los apoderados, e inclusive hacia sí mismos desde el discurso referido de los estudiantes. El corpus total analizado corresponde a 174 anotaciones. De ella se seleccionaron 33 ejemplos que se distribuyen en virtud de las tres dimensiones asociadas a los patrones valorativos encontrados.

Descripción de recursos valorativos

Destacaremos algunos recursos valorativos representativos de cada dimensión, considerando además la presencia de casos combinados dentro de una misma anotación. La organización de análisis incluirá anotaciones correspondientes a los parámetros de Juicio (J), Afecto (Af) y Apreciación (Ap). En cada caso se incluye primero si la anotación es positiva (+) o negativa (-), la sigla según el parámetro y el número de la anotación. La presentación se articula en torno a tres dimensiones relevantes, la primera referida a reglamentación de la permanencia que da cuenta de patrones que valoran personas, conductas, procesos y productos referidos a los rendimientos académicos, la disciplina, los procedimientos ante conductas y la toma de conocimiento del apoderado; la segunda referida a la presentación personal que da cuenta de patrones valorativos correspondientes al uso del uniforme escolar y al uso de accesorios, y la tercera, referida a la valoración de la labor académica docente que da cuenta de la colaboración de los alumnos en dicha acción.

I. Reglamentación de la permanencia:

\section{a) Conductas referidas a rendimiento académico}

(1)-J18 Se sorprende al alumno copiando durante el test de verbos.

(2)-J48 No trabaja en clases.

(3)-J87... no pone atención y se niega a salir a la pizarra a explicar contenidos recién expuestos. 
(5) +Af5+Af6 Felicitaciones Cristal Porque te has superado mucho (+Af5) en tus notas. Persevera. Sigue así (+Af6).

(6)+Af12+Af13 Se le ve más participativo y motivado (+Af12) a trabajar. Continúe así (+Af13).

(7)-Af15 Alumno no trabaja en clase, contando (Parafraseado incluido en el estilo indirecto) que no tiene interés para trabajar... (Alumno se evalúa a sí mismo)

(8)-Af16...No muestra actitud para dedicarse a sus estudios e insiste en no trabajar en clase.

(9)-Ap2 No entrega actividad y la deja sobre la mesa sin terminar.

\section{b) Conductas referidas a disciplina}

(10)+Ap21 Excelente comportamiento.

(11)-J68 No ingresa a la sala de clases. Llega con pase de inspectoría a las 13.30 horas, comenzando la clase a las 11.30 horas.

(12)+J111 Alumno que se compromete a mejorar su conducta y no tener ninguna anotación más.

(13)-Af18 Abandona dando un portazo al salir.

(14)-Af20-Af21 Rosa en reiteradas ocasiones, me contesta de muy mala forma, es atrevida (-Af20) y no le importa serlo delante del curso, a voz alta...Tiene actitudes agresivas (-Af21).

(15)-Ap4 Es enviado a inspectoría por pésima conducta.

\section{c) Procedimiento ante conductas}

(16)-Af24-Af25... Le llamo la atención, grita, la expulso a inspectoría, da portazo a la puerta.

(17)-J105 Alumno es suspendido de clases. Solamente puede y debe presentarse a rendir pruebas y exámenes.

(18)-J76 Alumno inasistente a clases; se procederá al cierre de su semestre con calificaciones Nota 2.0.

(19)-J78 Se le indica que abandone la sala y se niega ante lo cual se solicita a un alumno que vaya a inspectoría a solicitar la presencia en la sala de clases. 
(20)-J85 Lo envío a inspectoría por mala conducta. No trabaja solo conversa y pega con Andrade.

(21)-J101 Enviado a portería por agredirse con alumno Hugo Parra. (Inspector)

(22)-J103 El alumno es suspendido de clases por realizar actos reñidos con las mínimas normas de convivencia y respeto por los demás. (Inspector)

\section{d) Toma de conocimiento apoderado}

(23)-Ap10 El apoderado asiste y toma nota del comportamiento y rendimiento de su hijo. Además, sabe que tiene que buscar colegio para el próximo año. (incluye rendimiento y comportamiento).

(24)-J97 El apoderado toma conocimiento de las asignaturas insuficientes son Biología, Inglés, Historia, Química, Física, Matemática y con promedio 4.1.

(25)-J102 Se presenta apoderado y toma conocimiento de los atrasos de su pupila.

(26)-Ap9 El apoderado conoce los ramos insuficientes...

(27)-Ap15 Asiste su madre por estar involucrado en un hecho complicado. (Director)

En estos ejemplos podemos apreciar una mayor presencia de Juicios que en su mayoría son negativos y directos (2), (3), (11), no obstante, en la presentación de la situación al apoderado, el juicio de sanción social se presenta de forma evocada (24). Una característica relevante de esta dimensión de juicios, ya sea, en las conductas de rendimiento académico o de disciplina es la construcción adverbial negativa ante verbos de procesos materiales. En cuanto a las anotaciones positivas, estas se vinculan principalmente a afectos referidos a procesos conductuales con finalidad académica (5), y apreciaciones referidas a procesos conductuales (10), salvo la presencia de un juicio positivo (12) con cláusula introducida por conjunción subordinante y uso de "se" reflejo, más preposición e infinitivo. Por su parte, los afectos positivos son acompañados por una intensificación de afectos referidos a deseos de continuidad futura del proceso conductual celebrado (5) y (6) "Sigue así", "Continúe así". Los afectos negativos explícitos, al igual que parte de los juicios negativos, 
se construyen con adverbio de negación entre la agencia y el verbo. Por otro lado, las apreciaciones negativas están referidas a la calidad de los productos realizados por los estudiantes (9), (26), o bien, a hechos en que han participado (27).

Si bien la construcción prototípica de la anotación es por medio del presente de indicativo, con posibilidad de omitir la agencia y usar "se" impersonal. No obstante esta regularidad, fue posible identificar en el corpus analizado dos casos de personalización de docentes. En ellos los docentes dan cuenta de sus afectos (infelicidad) cuando la alumna encara a la profesora "me contesta"... "no le importa serlo delante del curso, a voz alta". Dicha situación se intensifica con el paso a la expresión de miedo de la docente al señalar "actitudes agresivas" de la estudiante. El otro caso (16) se plantea desde la acción valorativa de la docente y la reacción valorativa de la estudiante, constituyendo dos intercambios de cada lado. Esta situación expresada en la anotación permite intensificar la prosodia de afecto. La profesora le llama la atención, y la alumna grita, la profesora procede a la sanción, y la alumna manifiesta su insatisfacción de forma evocada dando un portazo.

En resumen el docente relata la conducta enjuiciándola, manifestando afecto o apreciándola, luego se expresan relatos de procedimientos sancionatorios que pueden o no contener las conductas que dieron paso al procedimiento, lo que involucra a este tercer tipo de anotación con la posibilidad de estar vinculada a más de un patrón valorativo. Finalmente, las anotaciones referidas a la toma de conocimiento por parte del apoderado vienen a sellar la validez de la anotación y fijan la relación y responsabilidad futura del establecimiento con el alumno. Por ello, se cita al apoderado como adulto responsable, ya que se informa de una sanción importante, pero no necesariamente grave como en el ejemplo (25), o bien, puede corresponder al fracaso escolar del alumno, en cuanto a que está en peligro de repitencia, o bien que realizó una conducta muy grave, o que en ausencia del alumno, el docente decide cerrar sus notas con una calificación determinada (18). También se le puede sancionar para que no asista más, ello antes del término del año escolar. Lo cual sólo autoriza al alumno a venir a rendir las pruebas pendientes (17), o derechamente se informa al apoderado que el alumno ya repitió el curso, o explícitamente se le informa al apoderado que no hay posibilidad de continuidad en el establecimiento para el año siguiente (23). En este sentido el proceso escolar en su conjunto presenta un input y un output. 


\section{Presentación personal:}

\section{a) Uso de uniforme escolar}

(28)-J38 Se presenta sin su uniforme completo. No tiene educación física y sin embargo asiste con pantalón de buzo, blusa y corbata.

\section{b) Uso de accesorios}

(29)-J26 Alumna viene maquillándose pese a ser advertida de no hacerlo.

En estos ejemplos que dan cuenta de la presentación personal se plantea desde el juicio explícito con la conjunción "sin", más una nominalización, o bien con un verboide o forma no personal en gerundio unido a una partícula refleja "se". En ambos casos se produce una intensificación de la prosodia valorativa, ya que se agrega mayor información. Dicha información constituye una valoración evocada. En el ejemplo (28) corresponde a un juicio de valor de desobediencia, a partir de una construcción negativa, por un lado, y por otro, se presenta una conjunción adversativa, "sin embargo". En el ejemplo (29) aparece otro intensificador adversativo, "pese a", que en otros casos figura como "a pesar de" o "a pesar que". En este último caso la anotación estipula dos valoraciones conjuntas, no obstante, estas operan como una única construcción retórica, en que lo más relevante no es la acción que contraviene las normas, sino el hecho que dicha acción se realiza aún cuando se emitió una advertencia anteriormente, por tanto la anotación en sí, no apunta a un hecho particular, sino que genera rasgos específicos de la personalidad de la estudiante en este caso, siendo una constante y no un hecho aislado.

En síntesis la anotación se produce para ser validada por sus lectores ideales, sirviendo de base para sostener decisiones académicas y/o disciplinarias al interior de la escuela, que regularmente implican sanciones sociales para el estudiante.

III. Valoración de la labor académica docente:

\section{a) Colaboración del/a estudiante}

(30)-J16 El curso conversa mucho, durante el transcurso de la clase, no prestan atención y se pierde bastante tiempo en mantener el orden para que nuevamente comiencen a conversar. 
(31)-J47 El alumno interrumpe la clase tirando papeles.

(32)-J69 Alumno golpea la pared molestando la clase y a la sala vecina.

(33)+Ap1 Muy buen trabajo en el diario mural.

Esta dimensión de patrones valorativos puede identificarse, ya sea con conductas referidas a rendimiento académico, o bien con conductas disciplinarias, dado que es temáticamente coincidente. No obstante, las valoraciones de esta dimensión se constituyen como juicios o apreciaciones que inciden en el desempeño del rol académico del docente, e impiden la realización esperada de la clase. Así, la valoración de juicio o de apreciación, como la de los ejemplos seleccionados, produce dificultades y/o beneficios para el desarrollo de la clase. Por ejemplo en el caso (30) en la cláusula "se pierde bastante tiempo" no aparece la agencia, que en definitiva es el propio profesor, quien pierde el tiempo para la realización de su clase, debido a las interrupciones de los alumnos. En este mismo ejemplo, se agregan intensificadores como el adverbio "nuevamente", que junto con señalar la reiteración de la conducta, del curso completo en este caso, da cuenta de la imposibilidad del docente para realizar la clase, y del mismo modo, pudiera estar evidenciando que el docente no logra manejar al grupo de estudiantes, al menos en cuanto al desarrollo académico disciplinar de la clase en cuestión. Lo mismo ocurre con los ejemplos (31) y (32). A su vez, y en consideración de la imposibilidad de manejo conductual de un alumno, de un grupo determinado, o del curso completo, este tipo de anotaciones exculpa al docente de no poder realizar la clase correspondiente, o bien pone en los alumnos la responsabilidad de no cumplir con el programa curricular.

\section{Conclusiones}

Los principales resultados del análisis pueden sintetizarse en las siguientes tablas que ilustran la distribución y presencia de las valoraciones desde el subsistema de actitud el cual considera los parámetros de juicio, afecto y apreciación.

\section{Distribución por parámetros de actitud}

Del total de anotaciones analizadas 111 corresponden al parámetro de juicio, 33 al de afecto y 30 al de apreciación. Los evaluadores son en su mayoría docentes, no obstante también figuran estudiantes evaluando 
afectos negativos hacia los profesores que los denuncian por medio de las anotaciones. A su vez, se da un caso aislado en que un docente evalúa a otro docente. En el caso de los juicios las personas evaluadas son en su totalidad estudiantes. En cuanto a las apreciaciones corresponden a productos académicos y situaciones conductuales. A nivel cuantitativo se presentan las figuras 1 y 2 que incluyen tablas totales y tablas específicas, respectivamente.

Las valoraciones positivas totales corresponden al 17,24\% y las negativas al 82,75\%. Del total de anotaciones el 81,6\% son evaluaciones directas y 18,39\% corresponden a evocaciones.

Las valoraciones negativas de juicio corresponden a un $89,18 \%$, y las positivas a un 10,81\%. Las valoraciones de afecto están más equiparadas con un $57,57 \%$ las negativas y un $42,42 \%$ las positivas. Las valoraciones negativas de apreciación presentan un $86,66 \%$ y un $13,33 \%$ las positivas. A nivel de parámetro específico se presentan las siguientes tablas en la figura 7.

\section{Figura $\mathrm{N}^{\circ} 6$}

\begin{tabular}{|c|c|c|c|}
\hline \multicolumn{4}{|c|}{ Distribución Total Valoraciones N=174 } \\
\hline+ & - & Dir & Evoc \\
\hline 30 & 144 & 142 & 32 \\
& & & \\
\hline $17,24 \%$ & $82,75 \%$ & $81,60 \%$ & $18,39 \%$ \\
\hline
\end{tabular}

\begin{tabular}{|c|c|l|l|}
\hline \multicolumn{4}{|c|}{ Juicio N=111 } \\
\hline+ & - & Dir & Evoc \\
& & & \\
\hline $10,81 \%$ & $89,18 \%$ & $80,18 \%$ & $19,81 \%$ \\
\hline
\end{tabular}

\begin{tabular}{|c|c|l|l|}
\hline \multicolumn{3}{|l|}{ Afecto $\mathbf{N}=33$} \\
\hline+ & - & Dir & Evoc \\
& & & \\
\hline $42,42 \%$ & $57,57 \%$ & $72,72 \%$ & $27,27 \%$ \\
& & & \\
\hline
\end{tabular}

\begin{tabular}{|c|c|l|l|}
\hline \multicolumn{3}{|c|}{ Apreciación N=30 } \\
\hline+ & - & Dir & Evoc \\
\hline $13,33 \%$ & $86,66 \%$ & $96,66 \%$ & $3,33 \%$ \\
& & & \\
\hline
\end{tabular}


A nivel de juicio, mayoritariamente se presentan sanciones sociales referidas a inadecuación y a falta de veracidad. El nivel de anotaciones positivas es muy bajo 12. Las valoraciones positivas totales corresponden al 17,24\% y las negativas al $82,75 \%$. Del total de anotaciones el $81,6 \%$ son evaluaciones directas y 18,39\% corresponden a evocaciones.

\section{Distribución por patrones valorativos}

I. Reglamentación de la permanencia:

\section{Figura $\mathrm{N}^{\circ} 7$}

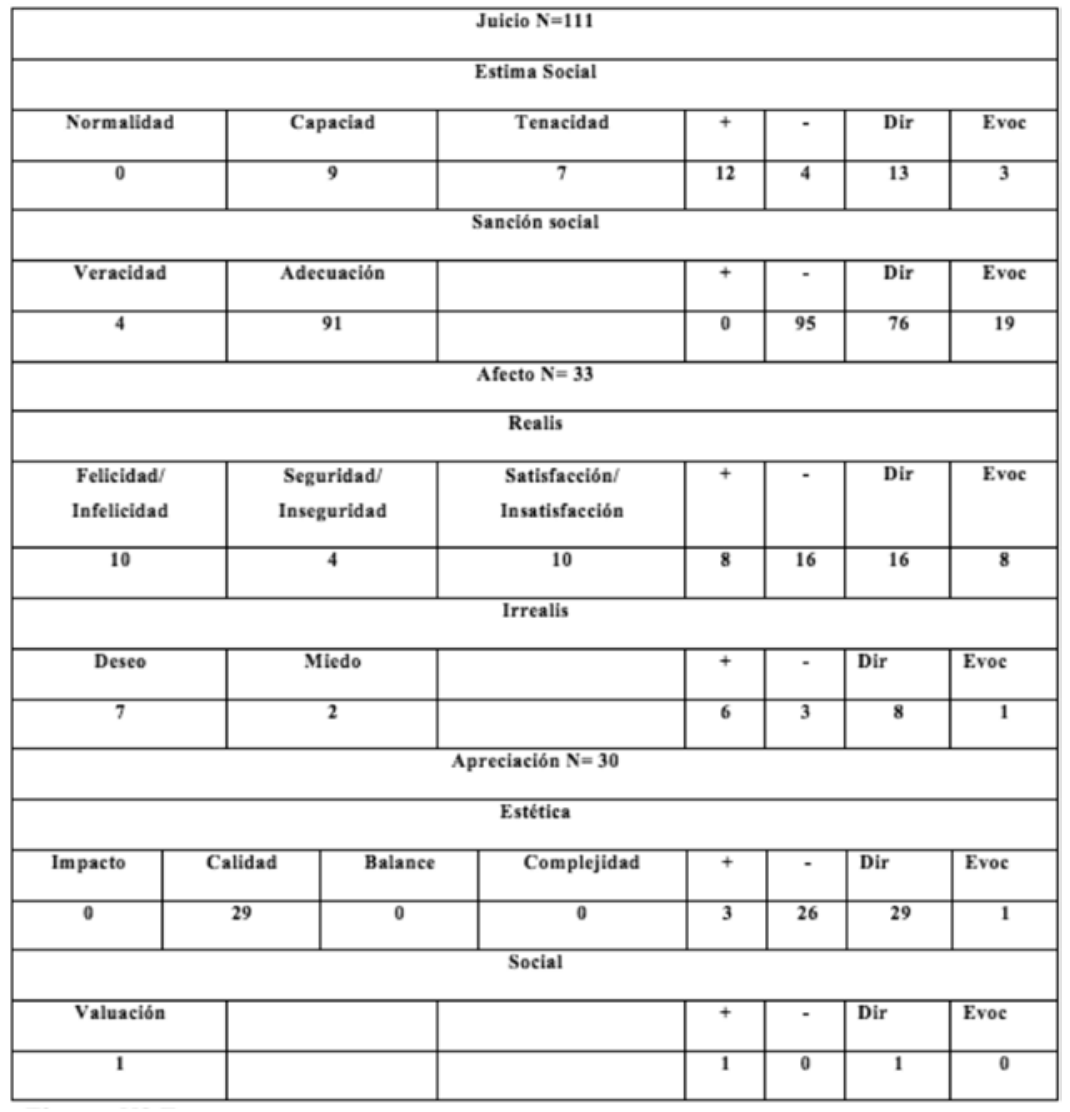

a) Conductas referidas a rendimiento académico

b) Conductas referidas a disciplina

c) Procedimiento ante conductas

d) Toma de conocimiento apoderado 
La construcción prototípica de la anotación es por medio del presente de indicativo, con posibilidad de omitir la agencia y uso de "se" impersonal. En cuanto a los juicios expresan principalmente de forma directa anotaciones negativas regularmente con adverbio de negación y asociado a verbos de procesos materiales. De igual forma los afectos negativos explícitos se construyen con adverbio de negación entre la agencia y el verbo. Por otro lado, las apreciaciones negativas están referidas a la calidad de los productos realizados por los estudiantes o bien, a hechos en que han participado.

Los juicios positivos pueden construirse con cláusula introducida por conjunción subordinante y uso de "se" reflejo, más preposición e infinitivo. Las positivas de afecto se construyen a partir de procesos conductuales con finalidad académica y apreciaciones referidas a procesos, a su vez tienden a estar acompañados de prosodia reiterativa de deseo de continuidad de la conducta felicitada.

En síntesis, la dimensión que constituye el patrón de la reglamentación de la permanencia da cuenta de un procedimiento sistemático y regulado, en el cual la anotación negativa en particular es su principal dispositivo que permite el ciclo del control del sistema normativo y académico del aparato escolar. En el input o entrada el docente relata la conducta enjuiciándola, manifestando afecto o apreciándola, luego se expresan relatos de procedimientos sancionatorios que pueden o no contener las conductas que dieron paso al procedimiento. Ello involucra un tercer tipo de anotación, que puede estar vinculado a más de un patrón valorativo a la vez, lo que evidencia la finalidad retórica del proceso. Finalmente, las anotaciones referidas a la toma de conocimiento por parte del apoderado vienen a sellar la validez de la anotación y fijan la relación y responsabilidad futura del establecimiento con el alumno. Por ello, se cita al apoderado como adulto responsable, ya que se informa de una sanción importante o grave, que puede corresponder al fracaso escolar del alumno, ya sea por suspensión definitiva, repitencia o cancelación de la matrícula para el año siguiente.

Las anotaciones cumplen el rol de evidenciar dicho tránsito en este sistema de control, a fin de justificar y validar los pasos que se deben realizar, hasta llegar incluso a la salida del alumno del establecimiento, lo que posiblemente en muchos casos pueda resultar finalmente, una salida definitiva del sistema escolar. Vulnerándose con ello los derechos de educación de estos jóvenes. 


\section{Presentación personal:}

\section{a) Uso de uniforme escolar}

\section{b) Uso de accesorios}

La intensificación de recursos de valoración en las anotaciones va instalando patrones actitudinales de los alumnos, con los que los profesores como productores esperan generar solidaridad ante sus lectores y/o destinatarios, a fin de que sus anotaciones sirvan de evidencia clara y contundente de los patrones conductuales. Todo ello para que los destinatarios superiores de la institución escolar como el inspector, el orientador, o el propio director, tomen decisiones asociadas a procesos sancionatorios. Y además, sirva de evidencia fidedigna al apoderado, para que éste último, quede convencido de la situación relatada en la anotación, y "tome conocimiento" del caso en cuestión. En suma, la anotación se escribe para que sea creíble y evidente ante cualquier lector y sirva de base para sustentar la decisión implementada a partir de esta, normalmente una sanción social con implicancias coercitivas para el estudiante.

\section{Valoración de la labor académica docente:}

\section{a) Colaboración del/a estudiante}

Esta dimensión se constituye como juicios o apreciaciones que repercuten en el desempeño del rol académico del docente, y que a su vez, impiden la realización de la clase. Así, la valoración de juicio o de apreciación produce dificultades y/o beneficios para el trabajo de aula. A su vez, y en consideración de la imposibilidad de manejo conductual de un alumno, de un grupo determinado, o del curso completo, este tipo de anotaciones exculpa al docente de no poder realizar la clase correspondiente, o bien pone en los alumnos la responsabilidad de no cumplir con el programa curricular. De cualquier forma, esta situación puede ocurrir incluso con un docente que tenga un buen manejo de un grupo curso. No obstante los alumnos realicen acciones específicas para interrumpir la clase y no presten atención al desarrollo de esta. Lo cual puede dar cuenta de la diferencia de metas entre el docente y los alumnos, y por cierto de la falta de expectativas, todo ello asociado a que las anotaciones analizadas en este estudio fueron recogidas dentro de un contexto vulnerable, por tanto, parte de las características propias de dicho contexto 
es justamente esta dificultad que impide poder desarrollar y desplegar el curriculum escolar de forma íntegra. Más aún, la dificultad del docente para lograr motivar a los alumnos a fin de desarrollar la clase.

\section{Referencias bibliográficas}

Achugar, M. (2008). What we remember: the construction of military memory in military discourse. Amsterdam: John Benjamins Publishing Company.

Alarcón, M. (2011). Aproximaciones al concepto de Discurso Profesional Docente. Literatura y Lingüística, 23, 141-165. http: / / dx.doi.org/10.4067/S0716-58112011000100009

Casas, L. Ahumada, C., Ramos, L., y Guajardo, A. (2008). La convivencia escolar, componente indispensable del derecho a la educación. Justicia y derechos del niño, 10, UDP UNICEF.

Chamorro, D., Barletta, N., \& Mizuno, J. (2013). El lenguaje para enseñar y aprender las Ciencias Naturales: Un caso de oportunidades perdidas para la formación ciudadana. Revista signos, 46 (81), 3-28. http://dx.doi. org / 10.4067/S0718-09342013000100001

Eggins, S. (2002). Introducción a la lingüística sistémica. Logroño: Servicio de Publicaciones Universidad de la Rioja.

Fairclough, N. (1995). Critical Discourse Analysis. The Critical Study of Language. London: Logman.

. (2003). Analysing Discourse. Textual analysis for social research. London/ New York: Routledge.

y Ruth, W. (1997). Critical Discourse Analysis. En T. van Dijk. (Ed.), Discourse as Social Interaction. London: Sage.

Gonzales, M. (2012). Teorías implícitas de docents del area de comunicación acerca de la comprensión lectora. Pontificia Universidad Católica de Lima. Tesis para optar al grado de Magíster en Educación con mención en currículo. 
Halliday, M. ([1982], 1994). El lenguaje como semiótica social. Bogotá: FCE.

. ([1985], 1994). An Introduction to Functional Grammar. Londres: Edward Arnol

y Hasan, R. (1989). Language, Context, and Text: Aspects of Language in a Social-Semiotic Perspective. Londres: Oxford University Press.

Kaplan, N. (2004). Nuevos desarrollos en el estudio de la evaluación en el lenguaje: la teoría de la valoración. Boletín de Lingüística (pp. 52-78). Caracas: Universidad Central de Venezuela.

. (2007). La teoría de la valoración: un desarrollo de los estudios sobre la evaluación en el lenguaje. En A. Bolívar (Coord.). Análisis del discurso. ¿Por qué y para qué? (pp. 63-86). Caracas: Universidad Central.

Martin, J. (2000). Beyond exchange: Appraisal systems in English. En Susan Hunston y Geoff Thompson (Eds.), Evaluation in text: Authorial stance and the construction of discourse (pp.142-175). Oxford: Oxford University Press.

y Rose, D. (2003). Working with discourse: Meaning beyond the clause. London: Continuum.

y White, P. (2005). The language of evaluation: Appraisal in English. Hampshire y New York: Palgrave Macmillan.

Moyano, E. (2010). Aportes del análisis de género y discurso a los procesos de enseñanza y aprendizaje escolares: las ciencias biológicas y la historia. Discurso y Sociedad, 4 (2), 294-331.

O'Donnell, Mick. (2013). UAM CorpusTool. CorpusTool is available from: http: / / www.wagsoft.com/CorpusTool/

Oteíza, T. (2006). El discurso pedagógico de la historia. Un análisis lingüístico sobre la construcción ideológica de la historia de Chile (1970-2001). Santiago, Chile: Frasis editores. 
. (2009). Solidaridad ideológica en el discurso de la historia: tensión entre orientaciones monoglósicas y heteroglósicas. Revista Signos, 42 (70), 219-244.

Román, M. (2006). El desafío de aprender a leer, comprender y razonar en escuelas vulnerables. Pensamiento Educativo, 39, 69-86.

Saussure, Ferdinand . D. E. (1945[1916]). Curso de Lingüística General. Madrid: Losada.

Sinclair, J. y Coulthard, M. (1975). Towards an analysis of discourse. Cambridge: Cambridge University Press.

White, Peter. (1998). Telling Media Tales: The News Story as Rhetoric. PhD. Thesis, Sydney: University of Sydney.

. (2000). Dialogue and inter-subjectivity: Reinterpreting the semantics of modality and hedging. En Malcolm Coulthard, Janet Cotterill y Frances Rock (Eds.), Dialogue analysis VII: Working with dialogue. Selected papers from the 7th International Association of Dialogue Analysis Conference Birmingham (1999) (pp. 67-80). Tübingen: Neimeyer.

. (2004). The Appraisal website: The language of attitude, arguability and interpersonal positioning. Disponible en: http:/ / www.grammatics.com/appraisal/index.html 